\title{
Transposition
}

Musique et Sciences Sociales

\section{Amateurs et professionnels, gratuité et profits aux premiers temps du Web 2.0 : l'exemple de la blogosphère musicale}

\section{François Ribac}

\section{OpenEdition}

\section{Journals}

\section{Édition électronique}

URL : http://journals.openedition.org/transposition/2483

DOI : 10.4000/transposition.2483

ISSN : 2110-6134

Éditeur

CRAL - Centre de recherche sur les arts et le langage

\section{Référence électronique}

François Ribac, «Amateurs et professionnels, gratuité et profits aux premiers temps du Web 2.0

l'exemple de la blogosphère musicale », Transposition [En ligne], 7 | 2018, mis en ligne le 15 septembre

2018, consulté le 06 mai 2019. URL : http://journals.openedition.org/transposition/2483 ; DOI :

10.4000/transposition.2483

Ce document a été généré automatiquement le 6 mai 2019.

\section{(c) (†) (?)}

La revue Transposition est mise à disposition selon les termes de la Licence Creative Commons Attribution - Partage dans les Mêmes Conditions 4.0 International. 


\title{
Amateurs et professionnels, gratuité et profits aux premiers temps du Web 2.0 : l'exemple de la blogosphère musicale
}

\author{
François Ribac
}

\section{La constitution d'un nouveau paysage}

\section{Élasticité des fichiers et inventivité des usagers}

À partir du milieu des années 90, le CD s'est peu à peu imposé comme le principal support d'écoute dans les pays industrialisés. Basé sur le fait de coder numériquement un enregistrement et de reconvertir le fichier en son lors de la lecture, ce format a été présenté par les fabricants d'appareils et de supports, les éditeurs, les majors et nombre d'états comme un progrès majeur, tant du point de vue de la qualité sonore que de la solidité des supports. Si la "révolution numérique » a contraint les consommateurs à remplacer leurs systèmes d'écoute et leurs vinyles, elle s'est également traduite par des effets imprévus que ses apôtres n'avaient pas anticipés et en particulier en matière de copie. En effet, s'il était courant de copier de la musique pour son entourage sur des cassettes puis des $\mathrm{CD}$ depuis la fin des années 60, les systèmes de peer to peer (P2P) ont soudain permis aux internautes d'échanger à volonté des fichiers mp3 et de les diffuser à une échelle jusqu'alors inédite. Conçu bénévolement par des informaticiens, le P2P a fortement fragilisé le monopole détenu par les distributeurs et les producteurs de musique enregistrée, affectant non seulement l'économie de la musique enregistrée, mais aussi les bases matérielles de la propriété intellectuelle. 


\section{Du côté de la prescription}

2 Le processus décrit ci-dessus a également son pendant du côté de la prescription musicale. Dès ses débuts, la toile a vu fleurir groupes de discussions, listes de news puis sites Web personnels consacrés à la musique. Mais il s'agissait uniquement (d'échanges) de textes. Les réseaux de $\mathrm{P} 2 \mathrm{P}$, dont certains étaient plutôt dédiés à des niches musicales ${ }^{1}$, ont constitué une première forme de prescription musicale à grande échelle, mais plutôt anonyme. Mais, l'apparition du Web 2.0 et de son cortège d'outils collaboratifs (blogs, forums, sites de partages de contenus, réseaux sociaux, sites de téléchargement, etc.) ont permis aux formes amateures de prescription d'étendre considérablement leurs déclinaisons, leur périmètre et leur visibilité. À tous les endroits de la toile, des amateurs émettent désormais des jugements sur la qualité esthétique des œuvres anciennes et récentes, patrimonialisent la musique enregistrée, écrivent des live report, éditent des newletters sur l'actualité du mainstream ou de minuscules niches stylistiques, etc. Parfois dotées d'audiences impressionnantes, ces plates-formes investissent de fait le territoire et la rythmique de la prescription professionnelle pour une rémunération essentiellement symbolique; un like, des remerciements, une citation dans la presse, la reconnaissance d'autres pairs. Comme pour l'industrie des supports et de la production musicale, le monopole des médias et des critiques musicaux professionnels (journalistes, académiques, programmateurs/trices, acteurs des politiques culturelles) est donc battu en brèche.

\section{Focus sur la blogosphère musicale}

Dans cet article, je voudrais précisément rendre compte de l'activité de certain-e-s de ces critiques de musique amateurs, animateurs/trices de blogs, webzines et forums et de leur influence non seulement sur le Web, mais aussi dans le monde professionnel des labels et des spectacles en Ile-de- France. Pour cela, je m'appuierai sur une enquête que j'ai menée de 2008 à 2010 en Île-de-France et sur le Web et qui portait sur l'activité de ces platesformes et de leurs animateurs/trices ${ }^{2}$. Un terrain constitué d'une trentaine de sites dédiés à la musique où la plupart des styles étaient représentés (du métal au classique en passant par le jazz) $)^{3}$. L'enquête datant désormais d'un peu moins d'une dizaine d'années, certains (f)acteurs ont perdu de l'influence (notamment les blogs) voire disparu alors que d'autres ont pris une plus grande importance (par exemple les réseaux sociaux). Cependant, cette période d'éclosion n'en est pas moins intéressante en ce sens qu'elle permet d'examiner comment cette prescription s'est mise en place, ce qu'elle a modifié dans le monde musical et pourquoi elle semble s'être pérennisée.

\section{Le terrain}

4 Je me suis tout d'abord intéressé à des blogs animés par une personne unique, publiant des articles (on dit des posts) signés avec son prénom et/ou son nom de famille ou - beaucoup plus fréquemment - un pseudonyme. Sur ces sites, les personnes contributrices exprimaient leurs goûts en se référant à leur érudition et à diverses sources extérieures, mais aussi à leur histoire personnelle ${ }^{4}$. 
5 Ensuite des webzines, des sites animés par plusieurs personnes et, qui de par leur organisation interne, leur mise en page, leurs choix éditoriaux et leur rythmique, s'apparentaient à des sortes de magazines culturels. Par extension, j'appelle blogzines, des blogs individuels dont le rythme de publication et la physionomie étaient comparables.

6 Enfin, des forums de discussion et/ou d'échange de fichiers musicaux où il fallait le plus souvent s'inscrire (voire participer un minimum) pour prendre connaissance des échanges et accéder à de la musique

7 La plupart des sites du panel proposaient d'écouter de courts extraits des musiques dont ils parlaient, par exemple en pointant vers des sites de streaming type Spotify ou Deezer ou de vidéo comme Youtube ou Dailymotion. Un des sites réalisait lui-même des vidéos. Mais certains (blogs mp3 et forums de discussion), minoritaires, distribuaient et échangeaient plus ou moins ouvertement des liens permettant de télécharger de la musique.

Dans cet article, je m'intéresse essentiellement aux blogs et aux webzines.

\section{Le panel}

9 La plupart des enquêté-es résidaient en Île-de-France et animaient bénévolement ces sites. Ils/elles ne tiraient pas - ou très marginalement - de revenus de leur(s) activité(s). À l'exception d'un blogueur rédigeant ses posts en anglais, tout le monde s'exprimait en français même si l'anglais pouvait être ponctuellement utilisé dans des discussions avec des visiteurs/trices.

Pour établir le panel, je me suis tout d'abord fié à ma propre connaissance de la blogosphère musicale francophone, ayant participé en 2006 à un blog mp3 rédigé en français. Mais j'ai également utilisé d'autres ressources comme la plate-forme internationale Totally Fuzzy qui recensait l'activité de nombre de blog mp3. Pour les autres types de sites, j'ai croisé plusieurs méthodes. J'ai testé le nombre d'entrées que Google accordait à ceux que j'avais repéré, recoupé leurs blogrolls ${ }^{5}$ et également utilisé les palmarès de Wikio (un site qui classait les blogs en fonction de leur citation sur la toile). Puis, au fur et à mesure que je menais des entretiens et que mon enquête était commentée dans la blogosphère musicale, on m'indiquait tel-l-e ou tel-le dont la réputation m'avait échappée. Si le panel comprend des plates-formes qui semblaient avoir acquis une certaine réputation, j'ai également veillé à ce qui figurent des sites moins « rayonnants » dédiés à de petites niches stylistiques. Au final, le panel était composé de 32 sites et de 26 personnes comprenant 21 hommes et 5 femmes (voir tableau 1 ). On remarquera que la plupart des personnes étaient dotées d'un emploi et que nombre travaillaient dans des secteurs dont les thématiques pouvaient se rapprocher du contenu de leurs blogs ou des compétences qui leur sont associées: les médias, la musique, le graphisme, l'informatique. 


\begin{tabular}{|c|c|c|c|c|}
\hline Résidence en IDF & Personne interrogée & Type de site & âge & Profession \\
\hline Paris $19 \mathrm{e}$ & Krüger & blog album & 50 & Informaticien \\
\hline Thaïlande & Laurent & blog album & 33 & Web designer \\
\hline Seine-Saint-Denis (93) & Francis & blog album & 38 & Relations publiques \\
\hline Paris $19 \mathrm{e}$ & Pierre & blog album & 26 & Illustrateur musical \\
\hline " & Pierre & blog album & “ & “ \\
\hline “ & Pierre & blog album & “ & " \\
\hline ville moyenne & Duno & blog album & 60 & Cadre commercial \\
\hline Meudon (92) & Alexandre & blogzine & “ & “ \\
\hline Paris $3 \mathrm{e}$ & Franck & blogzine & 35 & Archiviste \\
\hline Paris $11 \mathrm{e}$ & Nina & blogzine & 20 & Étudiante \\
\hline Paris $18 \mathrm{e}$ & Jonas & blogzine & 39 & Éditeur free lance \\
\hline Paris 11e & Poc & blogzine & 36 & Musicologue \\
\hline Asnières (92) & Patrick & blogzine & 28 & Cadre commercial à la Poste \\
\hline Paris $18 \mathrm{e}$ & Patrick & blogzine & “ & “ \\
\hline " & Carmen & blogzine & 28 & Relations publiques \\
\hline Paris $18 \mathrm{e}$ & Carl & blogzine & 37 & Consultant en informatique \\
\hline Lille & Isabelle & blogzine & 24 & Chargée projet environnement \\
\hline Asnières (92) & Michel & blogzine & 49 & Responsable de marché La Poste \\
\hline Aix en Provence & Veza & blogzine & 21 & Étudiante \\
\hline Levallois (92) & Scratch & blogzine & 36 & Sociologue \\
\hline “ & Luc & blogzine & 49 & Contrôleur de gestion \\
\hline Épinay sur Seine (93) & Pénélope & blogzine & 25 & Relations publiques \\
\hline Paris & Rolf & webzine & 37 & Responsable éditorial médias \\
\hline Paris $18 \mathrm{e}$ & Arnaud & webzine & 34 & Journaliste culturel \\
\hline Pré St Gervais (93) & Pénélope & webzine & 25 & Relations publiques \\
\hline Pré St Gervais (93) & Andy & webzine & 34 & Secrétaire de rédaction \\
\hline Clamart (92) & Mathias & webzine & 35 & Enseignant (musique) \\
\hline Sucie en Brie (94) & Jonas & webzine & 39 & Éditeur free lance \\
\hline Paris 11e & Alexandre & webzine & 30 & Dir. adjoint de magasin \\
\hline Paris $9 \mathrm{e}$ & Bertrand & forum/webzine & 46 & Auto entrepreneur/spectacles \\
\hline Achères (78) & Frédéric & forum & 49 & Collaborateur d'élus \\
\hline Paris $19 \mathrm{e}$ & Krüger & forum & 22 & Informaticien \\
\hline Le Mans & Kevin & forum/webzine & 23 & Journaliste sportif \\
\hline
\end{tabular}

\section{Thématiques}

11 Certains sites se consacraient exclusivement à un sujet, l'actualité musicale, un style spécifique, le patrimoine enregistré. Quelques-uns mélangeaient la musique avec la photo, le cinéma, le roman, etc. Pour ce qui concerne les styles musicaux, les sites du panel s'intéressaient surtout aux musiques populaires (rock, hip hop, techno, world music, musiques improvisées et expérimentales, chansons et leurs nombreux hybrides), mais plusieurs étaient dédiés à d'autres mondes : un webzine se consacrait à la musique classique, un autre s'intéressait régulièrement à la musique contemporaine, un blog d'albums de jazz et deux blogs d'albums à dominante rock parlaient régulièrement de musique classique ou contemporaine.

\section{Trois familles}

12 Au fur et à mesure que l'enquête avançait, j'ai de plus en plus distingué trois groupes : les réseauteurs/euses, les alternatifs et les indépendant-e-s. Pour définir et différencier ces idéaux-types, j'ai principalement pris en compte la façon dont les personnes et/ou les collectifs construisaient leur réputation, notamment leur relation aux internautes et les choses à propos desquelles ils et elles publiaient (ou pas). Je n'ai donc pas considéré que les différences stylistiques constituaient des critères déterminants pour établir cette typologie, car l'éthique et la pratique d'un blogueur spécialiste de rock pouvaient avoir beaucoup plus de proximités avec le responsable d'un webzine classique qu'avec l'animateur d'un blog mp3 consacré au rock. Pour les mêmes raisons, j'ai mis sur le même plan des plateformes individuelles et collectives? 


\section{Les réseauteurs (euses)} des objets et des évènements liés à l'actualité culturelle, au tempo du monde professionnel : sorties récentes de disques, tournées de formations musicales, parutions récentes sur la musique, etc. Dans ce groupe, on réalisait couramment des interviews, des reportages photo voire des vidéos d'artistes. Certains de ces sites proposaient des services aux internautes tels que des calendriers de concerts dans la capitale, des newsletters incluant aussi bien le sommaire du webzine que des événements artistiques à ne pas manquer ou encore des places de concerts à gagner. Une variante consistait à compléter la couverture de l'actualité par des articles à tonalité encyclopédique à propos d'artistes ou de courants musicaux. Les formes éditoriales typiques de la presse papier étaient parfois avec des innovations significatives - déclinées par plusieurs de ces sites. Certains se positionnaient explicitement comme de nouveaux acteurs en compétition avec la presse professionnelle.

D'une façon générale, les personnes de ce groupe publiaient beaucoup et régulièrement non seulement sur leurs sites, mais aussi sur d'autres blogs amis, les réseaux sociaux ( Twitter et Facebook) et veillaient constamment à ce que leur plate-forme soit bien référencée sur la toile. Pour prendre la mesure de cet activisme de tous les instants, notons qu'un des blogueurs du panel avait publié 1039 messages sur son site l'année précédant le début de l'enquête! Cette politique de visibilité se traduisait également par des liens tissés avec des labels de disques, des tourneurs ${ }^{8}$, des musées, des salles de spectacle, via leurs intermédiaires et/ou représentant-e-s; chargée-s de relations publiques, attaché-e-s de presse, agences de communication et jeunes agences de Web marketing dont les principales cibles étaient justement les blogs. Ces agences fournissaient ces blogs et webzines en informations (parfois exclusives), disques, films et livres. Elles organisaient également des rencontres avec des artistes ou des responsables artistiques. Elles choisissaient les blogs en fonction de leur classement par Wikio et si leur contenu éditorial leur semblait compatible avec les attentes de leurs clients. En échange de ces informations et services, les réseauteurs s'inséraient dans le dispositif de communication de leurs partenaires. Au moment où j'ai mené l'enquête, un des représentants les plus actifs de ce groupe (Patrick) organisait des rencontres où des professionnels-l-e-s de la musique et des blogueurs et blogueuses se retrouvaient pour boire un verre à Paris. Ce même blogueur animait le site d'une marque d'alcool qui sponsorise des concerts. Plusieurs de ces réseauteurs utilisaient leurs véritables noms et prénoms.

\section{Les alternatifs}

Si on les compare aux précédents, les alternatifs avaient pour première caractéristique de s'occuper principalement de rock et même de rock indé, au sens du courant musical et du type de critique journalistique nés à la faveur des mouvements punk et new wave à la fin des années 1970 (Cf. le magazine Les Inrocks). En termes d'écriture, leur style faisait souvent référence à la littérature et à des auteurs rock tels que Nick Tosches où l'érudition se combine souvent avec l'autobiographie.

Transposition, 7 | 2018 
La plupart des alternatifs utilisaient un pseudonyme et refusaient presque systématiquement d'être démarché-es par des labels, agences, représentants d'artistes. De façon concomitante, leurs posts et playlists étaient principalement dédiés à des répertoires enregistrés antérieurs et même si plusieurs allaient assister à des concerts, ils et elles ne les chroniquaient (pratiquement) pas et évoquaient peu les nouveautés. Néanmoins, les alternatifs travaillaient aussi à leur réputation. Ils/elles publiaient beaucoup et régulièrement, discutaient avec leurs pairs sur d'autres blogs et sur les réseaux sociaux, dialoguaient avec les internautes, ne manquaient pas de copier des liens ici et là, s'inscrivaient sur les sites de référencement, surveillaient leurs statistiques de fréquentation, etc. Lorsque la presse nationale les citait (par exemple sur la qualité des remasterings en mono des Beatles) ou les sollicitait, ils/elles ne se dérobaient pas. Simplement, leur éthique n'était pas compatible avec le fait de collaborer avec les différentes déclinaisons de l'industrie musicale et des politiques culturelles.

Le tableau $\mathrm{n}^{\circ} 2$ ci-après représente en trois séquences (préparation, rédaction, dialogues) les nombreuses activités auxquelles se livraient les réseauteurs et les alternatifs; veille, mise en page et écriture, échanges avec les visiteurs et les pairs, conversations sur les réseaux sociaux, dialogues avec des professionnels, etc. Un véritable travail!

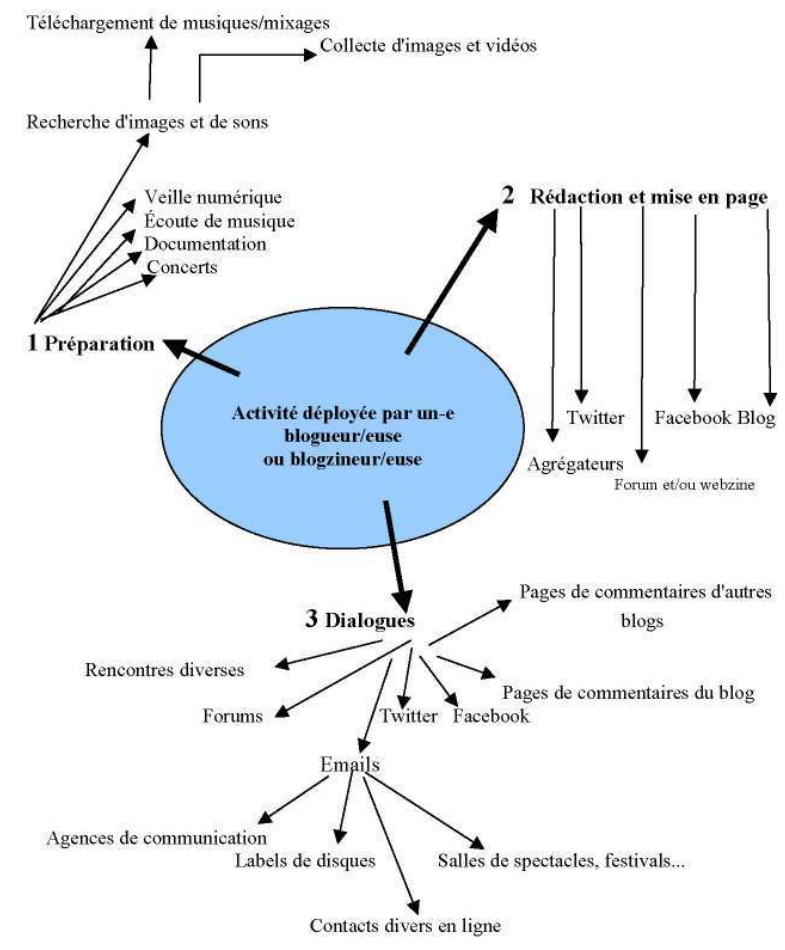

Tableau n 2 : I'activité d'un blog musical

\section{Les indépendant-e-s}

La caractéristique essentielle de ce groupe, composé de blogs mp3 et de forums de discussion, était de prescrire pour l'essentiel des enregistrements et d'accompagner ses critiques de liens permettant de télécharger des albums complets. Dans un contexte de mise en place de l'Hadopi', les indépendant-e-s bloguaient sans exception sous pseudonymes, n'avaient aucune relation avec des professionnels ou des labels de disques sauf quand ceux-ci leur ordonnaient de cesser de partager leurs disques. Les 
indépendant-es ne s'inscrivaient pas non plus sur les sites de référencement et de classement des blogs et ne s'exprimaient que dans les pages de commentaires de blogs du même type ou dans des forums fermés. Leur réputation pouvait donc seulement se déployer dans ce monde spécifique, peuplé de passionné-e-s à la recherche de disques rares introuvables ou (considérés comme) négligés. Écoutons par exemple Pierre qui pilotait pas moins de trois blogs consacrés à la library: B.O de films, "musiques au mètre » réalisées pour des films commerciaux ou d'entreprises, musiques électroniques des sixties et seventies.

Pierre : Concernant mes motivations : rendre à la sphère des music blogs ce qu'elle m'a donné... Aller sur des blogs que vous aimez, découvrir de nouvelles choses qui vous feront découvrir de nouvelles choses... La plupart des blogs que j'adorais n'existent plus, j'essaie de faire la roue de secours... Rendre hommage à la musique inconnue, me dire que grâce à moi, un album « revit » et est apprécié par trois cents personnes dans seize pays. J'aime bien. Être prescripteur de tendances, mettre un truc et simplement dire: " écoutez-moi ça les chéris »... Être un peu la contreculture (...). Proposer des alternatives et mettre en valeur le fait que ces alternatives étaient là depuis trente ans, sous votre nez, et qu'en fouillant un peu, on trouve des trucs super.

Pour autant, cela ne signifie pas que tous les indépendant-es ne travaillaient pas à leur renommée et publiaient en dilettante. Ainsi, des mesures effectuées avec le logiciel Issue Crawler $^{10}$ ont montré que d'avril 2007 à avril 2010, un blog mp3 de jazz du panel avait mis en ligne rien moins que 260 liens de téléchargement, chaque lien étant accompagné d'une chronique du disque (tableau $\mathrm{n}^{\circ} 3$ ci-après).

De leur côté, les internautes pouvaient évaluer la réputation des blogs mp3 en se référant aux compteurs qui référençaient le nombre de visiteurs et/ou de téléchargements ou encore au nombre et au contenu des commentaires déposés sur un blog. À cette reconnaissance "publique » s'ajoutait une reconnaissance plus discrète : amitiés nouées via de messages privés dans les forums ou la plate-forme de P2P Soulseek, réception de rips $^{11}$ de vinyles souvent introuvables et dont la valeur marchande pouvait être considérable.

Le tableau ci-dessous répertorie les disques proposés par Duno dans son blog d'album à dominante jazz Swinglow d'avril 2007 à avril 2010. Chaque ligne inclut le jour de publication de la critique et du lien de téléchargement, le nom de l'artiste et/ou de la formation, le nom de l'album et le type de compression du format mp3. On voit ainsi apparaitre la carrière de ce blog et le type de contenus proposés sur une longue période. Les albums postés plusieurs fois ne sont pas recensés ici. On notera que la quasi-totalité des disques proposés sont des disques anciens (voire introuvables). 
12007/04/beatlejazz-bite-of-apple-2356-kbps

007/04/branford-marsalis-quartet-requiem-320

2007/04/esbjrn-svensson-trio-tuesday-wond
2007/04/jacques-loussier-pulsion-192-kbps

2007/04/jon-vangelis-short-stories-vbr.htm

2007/04/keith-jarrett-kln-concert-192-kbps

2007/04/kornet-fritt-fall-224-kbps

2007/04/nguyn-l-duos-homescape-vbr

2007/04/pat-metheny-group-offramp-vbr

2007/04/qui-aurait-pari-il-y-quelques-annes-que

12007/04/skalpel-skalpel-320-kbps

2007/04/stephane-grappelli-claude-bolling-first

2007/05/aretha-franklin-amazing-grace-2-cd-32

2007/05/billie-holiday-retrospectives-1935-1952

2007/05/camille-bazbaz-une-envie-de-chien-192

2007/05/eric-legnini-miss-soul-192-kbps

2007/05/john-zorn-stone-issue-one-128-kbps

2007/05/kenny-barron-quintet-images-320-kbp

2007/05/khalit-chahine-mektoub-320-kbps

2007/05/klaus-schulze-pete-namlook-dark-side-of

12007/05/maurice-wander-chess-256-kbps

/2007/05/mile-davis-nefertiti-320-kbps

2007/05/neil-young-crazy-horse-reacto

2007/05/neil-young-harvest-128-kbps

2007/05/pierre-de-bethmann-complexe-256-kbps

2007/05/santana-lotus-gem-320-kbps

/2007/05/amy-winchouse-franck-192-kbss

2007/06/count-basie-orchestra-live-at-el

2007/06/christian-vander-trio-jour-aprs-jour

12007/06/goran-kajfes-head-spin-224-kbps

/2007/06/john-coltrane-quartet-ballads-192-kbps

/2007/06/joshua-redman-momentum-192-kbps

$12007 / 06 /$ pierre-bensusan-didier-malherbe-live-in

$12007 / 07 /$ bill-bruford-gradually-going-tornado

2007/07/charlie-parker-jam-session-320-kbp

2007//07/didier-malherbe-fluvius-128-khps

2007/07/edition-speciale-alicante-192-kb

2007/07/koenihihyakkei-angherr-shisspa-320-kbp

12007/07/koenji-hyakkei-hundred-sights-of-koenji

/2007/07/redshift-ii-ether-128-kbps

2007/07/redshift-i-redshift-192-kbps

2007/07/redshift-ix-toll-192-kbps

2007/07/redshift-v-halo-256-kbps

Tableau $n^{\circ} 3$

\section{De petites sociétés encastrées}

Dès les premiers entretiens avec les réseauteurs et les alternatifs, je n'ai pas tardé à me rendre compte que le concept de blogosphère musicale francilienne était bien réel. En effet, la plupart des personnes que j'avais contactées se connaissaient et pour certaines se rencontraient régulièrement, tant sur la toile qu'à Paris. Les réseauteurs et les alternatifs étaient presque constamment connectés avec des pairs. On échangeait beaucoup via Twitter et Facebook, à l'époque en plein essor, où l'on utilisait les mêmes pseudos que sur les sites Web. Au moment où l'enquête a été réalisée, Facebook avait de plus en plus tendance à être utilisé pour répliquer et compléter les posts sur les blogs et les webzines.

Les échanges qui se déroulaient sur Twitter se déclinaient de bien des manières: des sortes de carnets de notes instantanés («impossible de dormir»), des infos sur ses déplacements («je suis au Trabendo»), des annonces de nouveaux posts («Bob Mould revient avec un nouveau disque »), des appels à se retrouver à Paris («qui va voir les Pixies ce soir?») des évènements que l'on organisait, des commentaires ironiques - mais jamais violents - envers des pairs et beaucoup de plaisanteries. Cette décontraction permettait de remiser au second plan les différences entre les réseauteurs et les alternatifs et la concurrence. Ce recours constant à l'humour et à l'auto dérision caractérise fortement ce monde qui, quoique passionné et méthodique, se définit comme amateur et où il est mal vu - tant du côté des internautes que des pairs - de se prendre par trop au sérieux

Du côté des réseauteurs, les discussions sur Twitter impliquaient régulièrement des professionnel-l-e-s et en particulier deux agences de Web marketing. Celles-ci m'ont d'ailleurs confirmé que ces échanges contribuaient à établir un climat de confiance. 
Quoi qu'il en soit, si l'on additionne l'ensemble des conversations en ligne, l'activité des sites et leur fréquentation, les rendez-vous entre pros et blogueurs (euses), les rencontres lors des spectacles et évènements initiés par des professionnels, les rencontres informelles dans les concerts, les bars et les expositions on se rend alors compte que la blogosphère francilienne de 2008/2010 constituait véritablement un petit groupe social. C'est à l'impact de cette blogosphère auquel je vais maintenant m'intéresser.

\section{Mesurer l'influence de la blogosphère}

\section{Une intégration partielle au monde professionnel}

26 J'ai déjà mentionné plus haut que les réseauteurs et quelques alternatifs entretenaient des liens avec le monde professionnel ; salles, festivals, musées, promoteurs de spectacles, presse papier et médias audiovisuels, majors et labels indépendants, professions dédiées à la promotion, à la communication et au marketing. Ainsi, lorsque j'ai débuté mon enquête, de nombreux partenariats étaient d'ores et déjà tissés entre des réseauteurs et des structures professionnelles. Nombre avaient accès à des informations concernant des artistes, des disques, des concerts, des expositions (par exemple celle consacrée à Miles Davis à la Cité de la Musique à Paris), des interviews, des pass dans des festivals, etc. Ils/ elles organisaient des quizz pour gagner des places, chroniquaient régulièrement les sorties d'un label, étaient associé-es avec des salles de spectacles ou des radios. Bien qu'achalandés en grande partie par des attachée-s de presse et des agences de Web marketing, les réseauteurs affirmaient qu'ils conservaient leur entière liberté de jugement.

Certains sites avaient réussi à s'imposer comme des prescripteurs à valeur ajoutée grâce à leur originalité éditoriale, comme ce webzine qui filmait des concerts en appartement de formations musicales de passage à Paris. Ce format avait tellement de succès auprès des internautes, des professionnels et des artistes que de nombreux labels et tourneurs s'efforçaient d'obtenir que leurs artistes soient filmés par l'équipe et figurent sur le webzine. Aujourd'hui, ce " produit » est véritablement devenu un standard dans le monde du rock. Au moment de l'enquête, des membres fondateurs de ce site assuraient partiellement la programmation d'une salle de spectacle parisienne privée. Certains alternatifs se produisaient régulièrement en tant que DJ dans des cafés parisiens. Plusieurs blogueuses (réseauteuses ou alternatives) effectuaient des piges en tant que chargée de relations publiques ou attachée de presse, notamment pour des petits labels de rock.

Du côté de la diffusion d'informations, plusieurs newsletters éditées par des webzines - et en particulier une produite par un site dédié à la musique classique et à l'opéra - étaient réalisées en collaboration avec de grandes institutions publiques, des festivals et des organismes de promotion de la musique classique. Cette "newsletter classique » était utilisée par les magazines professionnels et consultée par la profession. Quelques alternatifs étaient régulièrement cités par la presse nationale comme des experts crédibles, et discutaient en ligne - parfois quotidiennement - avec des journalistes musicaux professionnels, sans se départir pour autant de leur anonymat.

On l'aura compris, certaines de ces plates-formes avaient acquis une place dans l'industrie musicale (au sens le plus large du terme) et pour quelques-unes concurrençaient leurs " équivalents » professionnels. Elles étaient considérées comme 
des prescripteurs influents, capables d'innover et d'attirer du public et traités comme tels.

\section{Les agences de Web marketing} blogosphère. Elles s'activaient en effet auprès des majors du disque, des salles de concert et des institutions publiques pour faire valoir l'importance de ces nouveaux prescripteurs et la nécessité de travailler avec eux. Fans de musiques, très au fait des courants musicaux et des niches, fréquentant énormément les concerts, les responsables de ces agences prenaient en compte la sensibilité des animateurs/trices de sites, leur flair, la qualité de leur écriture. Pour convaincre leurs clients, elles faisaient d'abord valoir des arguments quantitatifs; les statistiques de fréquentation des sites, leur classement dans les sites de référencement, les mentions de ces blogs que l'on pouvait trouver sur le Web. Elles insistaient également sur l'importance pour leurs clients (artistes, labels, produits) de pouvoir compter sur des traces sur la toile. Or justement, comparés aux sites Web de la presse nationale - dont la plupart rendaient payants les articles quelques jours après leur parution - les blogs amateurs laissaient accessibles tous leurs contenus. Les agences de Web marketing avaient déjà compris que la réputation d'un produit culturel s'appuie non seulement sur le fait d'obtenir un bon papier dans un journal national, mais aussi sur le fait d'être abondamment référencé par les moteurs de recherche.

\section{Les territoires de la prescription}

31 Une mesure de la rézodience ${ }^{12}$ des sites du panel a permis de vérifier la consistance des liens entre les réseauteurs et les alternatifs et la sphère professionnelle à Paris et en Îlede-France. Dans un premier temps, le logiciel Issue Crawler a scanné les codes source ${ }^{13}$ des 26 sites du panel dont les animateurs/trices avaient des liens (résidence et/ou travail) avec l'île-de-France et qu'on appellera le noyau. Le logiciel a ensuite recensé toutes les pages Web citées au moins deux fois par ces mêmes 26 sites et a ainsi mis à jour les adresses URL ${ }^{14}$ de 81 sites que l'on appellera la périphérie. Le recensement des sites mentionnés au moins deux fois dans le "grand réseau " (noyau + périphérie) a fait apparaître les résultats suivants :

- 47 blogzines musicaux (dont 1 anglophone et 7 du panel)

- 11 sites généralistes (réseaux sociaux, partage de vidéos en ligne, Wikipedia, etc.)

- 11 sites d'artistes à visibilité internationale

- 3 labels de disques

- 9 lieux situés, à une exception près, en Île-de-France 


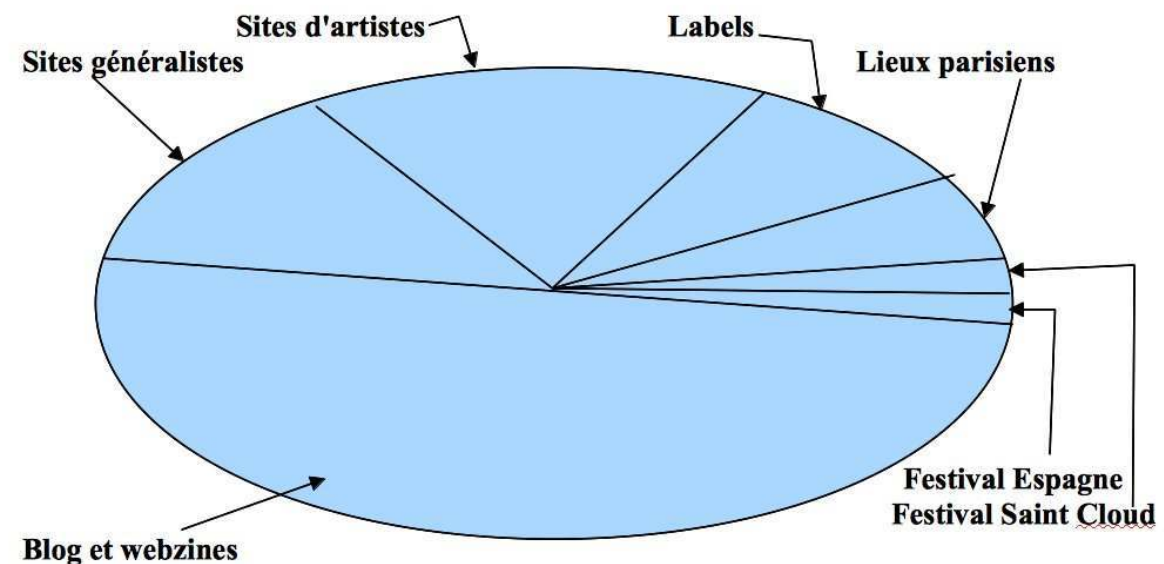

Tableau n 4 : Les différentes parties du « grand réseau »

Si l'on dresse la liste des lieux recensés à Paris et en Île-de-France cela donne :

\begin{tabular}{|l|l|l|}
\hline Nom du lieu & Activités & Ville \\
\hline Point Éphémère & Spectacles/Répétitions & Paris 10e arrondissement \\
\hline La Bellevilloise & Spectacles & Paris 20e arrondissement \\
\hline La Flèche d'or & Spectacles & Paris 20e arrondissement \\
\hline La Maroquinerie & Spectacles & Paris 20e arrondissement \\
\hline Le Glazart & Spectacles & Paris 19e arrondissement \\
\hline Fondation Cartier & Art Contemporain/Spectacles & Paris 14e arrondissement \\
\hline Groundzero & Disquaire & Paris 10e arrondissement \\
\hline Rock en Seine & Festival & Parc de Saint Cloud/Hauts de Seine \\
\hline FIB & Festival & Benicasim/Espagne \\
\hline
\end{tabular}

Plusieurs choses méritent d'être soulignées à cet endroit.

Premièrement, le grand réseau (noyau + périphérie) est en grande partie constitué de blogs et de webzines référencés par leurs pairs. Cela confirme l'idée qu'une communauté de sites consacrés à la musique, la blogosphère musicale, s'est bien formée.

Deuxièmement, l'effeuillage du grand réseau fait apparaitre 7 blogs du panel qui comprennent des réseauteurs et alternatifs hyper actifs du panel. Les indépendants (blogs ou forums) ne sont pas présents, ce qui confirme leur confinement aux marges. Autrement dit, si vous publiez beaucoup et régulièrement; que vos contenus sont jugés 
crédibles par vos pairs et des internautes; et que vous interagissez régulièrement avec d'autres usagers et visiteurs, votre réputation en ligne peut se développer et s'affermir ${ }^{15}$.

Troisièmement, $10 \%$ des liens du grand réseau pointent vers des lieux physiques dédiés aux spectacles, aux expositions (la Fondation Cartier) ou à la vente de disque, situés en Île-de-France, et en tout premier lieu à Paris. Ce résultat confirme les liens tissés entre des fractions de la blogosphère et le monde professionnel de la capitale. Par là, on voit les connexions entre une critique en ligne qui donne la part belle aux disques et le monde du live, en particulier des salles de spectacles privées qui programment du rock à Paris intramuros.

Quatrièmement, on voit que la centralité parisienne s'exerce aussi sur le Web. Là comme ailleurs en France, Paris capte l'attention des critiques amateurs, car pour eux aussi c'est là que les ressources et les événements décisifs se trouvent.

Enfin, on peut remarquer que ces liens avec le monde du spectacle en Île-de-France concernent essentiellement des dispositifs ne recevant pas ou peu de subventions publiques. Seul le Festival Rock en Seine, qui recevait à l'époque un fort soutien financier du département des Hauts de Seine, fait exception.

\section{La blogosphère, une activité rémunératrice?}

Compte tenu de l'insertion dans le monde professionnel de certains sites, le blogging pouvait-il constituer un chemin vers un emploi dans la presse professionnelle ou le monde du spectacle? On peut sérieusement en douter.

41 Tout d'abord parce que la presque la totalité du panel s'y refusait. Lors des entretiens j'ai été très frappé par l'insistance de la quasi-totalité des blogueurs/euses sur la dimension strictement amateur de leur pratique, et ce y compris chez les plus actifs des réseauteurs/ trices. Patrick dont le blog était classé en tête de Wikio, qui faisait des piges pour le blog musical d'une marque d'alcool et organisait des rencontres informelles avec des pros déclarait :

Patrick : Si je continue de vouloir organiser des événements fédérateurs au niveau de la blogosphère, (...) je veux surtout que cela reste une passion et m'éloigner le plus possible de toutes contraintes professionnelles. À bien des égards, j'essaye de me faire discret aujourd'hui. Ça doit rester un hobby, je ne suis pas journaliste professionnel et je ne veux pas devoir assumer une notoriété plus forte.

De même, les membres du panel qui travaillaient dans le monde de la musique ou des médias m'ont présenté leur blog ou webzine comme un espace où ils/elles pouvaient s'exprimer en toute liberté.

43 L'autre point qui fait douter de la possibilité que même les plus actifs de la blogosphère musicale basculent durablement et pleinement dans le monde professionnel tient à la modicité des rémunérations sur la toile. Ainsi, l'animatrice d'un blogzine a révélé il y a quelques années avoir été approchée par le magazine 20 ans pour effectuer des piges. Cette blogueuse dénonça non seulement les tarifs dérisoires qu'on lui proposait pour une page (il lui fallait écrire 132 pages par mois pour prétendre au smic), mais aussi le fait que l'on propose à des amateurs de concurrencer des journalistes professionnels ${ }^{16}$. Il semble encore en être de même à l'heure actuelle; pour dégager de véritables revenus, les Youtubeurs/euses doivent agglomérer des audiences considérables qui se chiffrent en centaines de milliers d'abonnés ${ }^{17}$. Autre exemple, les fondateurs de la plate-forme qui a imposé le format des concerts en appartement ont effectivement basculé vers le monde 
professionnel. Cependant, si le blogging a effectivement constitué pour eux un tremplin vers un emploi, cela a impliqué le naufrage partiel de leur site et de fortes dissensions. Cette exception semble donc confirmer la règle. En 2010, à l'issue de cette enquête, ni les réseauteurs, ni les alternatifs les plus actifs ne semblaient pouvoir obtenir une véritable rémunération de leur activité. Au mieux, le blogging pouvait constituer un sas.

\section{Et maintenant?}

Six ans après la fin de cette enquête, les plus actifs et les plus visibles des réseauteurs et des alternatifs sont encore en activité et, pour la plupart avec le même tempo soutenu. Toutes et tous ont intégré à leur pratique le fait de publier et d'interagir avec d'autres usagers sur les réseaux sociaux, Facebook, Twitter, mais aussi Instagram (et des équivalents). Deux d'entre eux ont écrit des livres, l'un avec son véritable nom (le réseauteur), l'autre sous pseudonyme (l'alternatif). Du côté des indépendants, si de nombreux blogs mp3 et un forum du panel ont disparu ou ont été abandonnés, là encore les plus actifs sont toujours présents.

\section{Pistes de réflexion}

Pour conclure la présentation de ce(s) monde(s), je voudrais mentionner quelques travaux qui, à mon sens, éclairent le surgissement et le maintien de la blogosphère musicale et sa contribution au monde musical.

\section{Comment la blogosphère musicale a-t-elle surgi ?}

La théorie de la longue traîne de Chris Anderson (2009) a montré qu'en s'affranchissant de nombre des contraintes et coûts propres aux magasins (personnel, loyer, fiscalité, situation, exposition des marchandises, etc.) les sites de vente en ligne peuvent alors non seulement stocker et vendre du mainstream à moindre prix, mais aussi des produits de niches qu'il leur aurait été impossible de conserver et d'exposer dans des magasins. Même si chaque produit de niche ne trouve qu'un acheteur par mois, l'accumulation des ventes de références isolées peut optimiser très fortement les profits. L'intérêt de l'approche d'Anderson est de montrer que si les consommateurs de l'avant Web se concentraient sur le mainstream c'est notamment parce qu'ils n'avaient que très peu accès à d'autres biens. Précisément, on peut comprendre la blogosphère musicale comme une sorte de «longue traîne prescriptive ». En s'appropriant les outils du Web 2.0, les animateurs/trices de la blogosphère musicale ont pu s'affranchir des contraintes matérielles de la critique professionnelle et de la presse ${ }^{18}$ et disposer d'un périmètre et d'une audience importante dont aucun fanzine des seventies n'aurait osé rêver. De ce fait, cette prescription a alors le loisir de parler tout autant du mainstream que des niches, de même qu'elle peut expérimenter toutes sortes de manières de prescrire.

\section{Pourquoi la blogosphère musicale a-t-elle surgi ?}

Dans L'économie des singularités (2007), Lucien Karpik rappelle qu'à la façon d'autres produits ou services (la haute couture, les prestations de avocats ou des architectes) les biens culturels se caractérisent par le fait d'être extrêmement difficiles à apprécier à 
l'avance. Un même spectacle peut être extrêmement différent selon les représentations, un artiste confirmé peut sortir un disque qui déconcerte ses fans, etc. Afin de réduire l'incertitude qui est inhérente à ces biens, les usagers se tournent donc vers des instances et des personnes qui les informent et leur garantissent un peu moins d'obscurité et un minimum de sécurité: institutions, organes de presse, médias, programmateurs, critiques, universitaires, ce que Karpik appelle des « dispositifs de jugement $»^{19}$. Si Karpik ne parle pas d'Internet, il est patent que les notes et les commentaires accompagnant les offres de produits culturels en ligne, les discussions sur les réseaux sociaux à propos de biens culturels et bien entendu les prescriptions de la blogosphère s'apparentent bien à des dispositifs de jugement ${ }^{20}$. La grammaire de Karpik nous suggère alors que la mise à disposition d'un nombre sans précédent d'œuvres musicales sur la toile (cf. la longue traîne) pourrait être corrélée avec l'accroissement et la diversification de l'expertise et des recommandations culturelles. Si la blogosphère musicale a trouvé et fidélisé ses divers publics, c'est probablement parce que sa pluralité permet aux internautes de mieux s'orienter et d'accéder à la musique à l'ère numérique. Et de même, la grande diversité des formes de prescription musicale coïncide avec l'infinie variété des genres et des goûts musicaux que l'effet de longue traîne rend visible.

\section{La question des motivations}

Pour comprendre le surgissement et la persistance de cette forme d'expertise, on peut également mobiliser une autre grille de lecture qui, plus encore que celle de Karpik, prend en compte les ressorts collectifs et sociaux. Dans Exit, voice and loyalty, (1970/2011), Albert Hirschman propose l'idée que lorsqu'un produit ou un distributeur domine un marché, une certaine fraction des consommateurs a tendance à s'en détourner. Par exemple, les acheteurs de nourriture bio se méfient des produits de ce type proposés par la grande distribution, car ils ne croient pas que cette dernière puisse respecter l'éthique et les normes du bio. Autrement dit, «l'effet mainstream» induit une méfiance et provoque la fuite d'une partie des consommateurs (exit). Une fraction de ces mécontentes peut être alors amenée à prendre la parole pour exprimer son indignation (voice), par exemple en créant une association de consommateurs ou une coopérative bio. Selon Hirschman, un troisième groupe continuera néanmoins à faire confiance à l'enseigne et/ ou à la marque, gage de sécurité et de stabilité (loyalty). Fort de cette perspective, on peut comprendre le public de la blogosphère musicale comme la partie des consommateurs qui se méfie de la critique musicale instituée (exit) et le blogging comme son porte-parole ( voice). Cependant qu'en parallèle à cette offre alternative et bénévole, une partie des consommateurs reste attachée à la critique et aux médias traditionnels et professionnels, parce leur durée de vie et leur taille leur semble constituer une assurance de sérieux et de qualité (loyalty) ${ }^{21}$. Et de la même manière que la blogosphère est bigarrée et partiellement en contact avec le monde professionnel, le public peut évidemment voyager d'un pôle à l'autre.

\section{Qui profite de la blogosphère?}

49 On l'a vu plus haut, le travail de la blogosphère musicale ne profite pas qu'aux internautes, il est aussi mobilisé, au moins en partie, par le monde professionnel. 

désigne ainsi les nombreuses activités auxquelles se livrent les internautes sur la toile, qu'il s'agisse par exemple d'interactions au sein des réseaux sociaux ou d'engagements plus formalisés comme animer un blog. Or, nombre de ces données, produites gratuitement par des personnes et/ou des collectifs, sont captées par des firmes pour les informer sur le profil des internautes et (tenter d')orienter les stratégies de ces firmes. En d'autres mots, l'activité des uns sert à enrichir quelques autres, sachant que cette expropriation, et son pendant la surveillance de nos activités, s'effectuent subrepticement. De la même manière, les activités foisonnantes de la blogosphère et des internautes qui la fréquentent sont pistées, analysées par des firmes et très certainement revendues à d'autres. Toutefois, l'enquête montre que le travail de la blogosphère n'est pas uniquement capté sur et pour le Web. On se rappelle que les composantes "spectacles» et «médias traditionnels » de l'industrie musicale - qui officient sur le territoire d'avant les réseaux électroniques - ont également recours à la blogosphère. De même, nombre d'informations, prescriptions, analyses et découvertes produites par la blogosphère sont citées (gratuitement) par la presse nationale et mobilisées par des agences de Web marketing. Et de fait, le travail de ces dernières consiste bien à monétiser (pour elles et leurs clients) ces activités bénévoles. Même la fraction de la blogosphère qui distribue illégalement de la musique contribue à ce transfert de valeur. Car, les innombrables raretés discographiques et les artistes oubliés que les indépendants extraient régulièrement du néant sont tôt ou tard repérés par des professionnels qui en cas d'engouement se chargent ensuite de rééditer les disques de ces artistes et, s'ils sont vivants, de leur organiser des tournées. En d'autres mots, le digital labor de la blogosphère musicale est mobilisé par toutes les composantes de l'industrie musicale et dans toutes sortes de territoires. On voit par-là que penser les mutations de l'économie de la musique implique de prendre en compte ce continuum entre le territoire «traditionnel» et le Web, d'une part, et entre amateurs et professionnels, d'autre part.

Plus généralement, le fait que cette expertise gratuite et bénévole ait trouvé son public et convaincu une partie du monde professionnel nous invite certainement à repenser l'égalité entre professionnalisme et qualité qui domine les jugements en matière de musique et d'art.

\section{BIBLIOGRAPHIE}

ALLARD Laurence, « Émergence des cultures expressives, d'Internet au mobile », Mediamorphoses, $\mathrm{n}$ o 21, 2007, p. 19-25.

ANDERSON Chris, La Longue traîne, Paris, Pearson, 2009.

BEUSCART Jean-Samuel, « Sociabilité en ligne, notoriété virtuelle et carrière artistique, les usages de MySpace par les musiciens autoproduits », Réseaux, nº 152, 2008, p. 139-168.

Transposition, 7| 2018 
BEUSCART Jean-Samuel, CARDON Dominique, PISSARD Nicolas, PRIEUR Christophe, « Pourquoi partager mes photos de vacances avec des inconnus, les usages de Flickr », Réseaux, n 54, 2009, p. 93-129.

BEUSCART Jean-Samuel et MELLET Kevin, « La conversion de la notoriété en ligne. Une étude des trajectoires de vidéastes pro-am », Terrains et Travaux, vol. 26, n 1, 2015, p 83 -104.

BOUQUILLON Philippe et MATTHEWS Jacob T., Le Web collaboratif. Mutations des industries de la culture et de la communication, Grenoble, Presses universitaires de Grenoble, 2010.

CARDON Dominique, « Le désign de la visibilité Un essai de cartographie du web 2.0 », Réseaux, $\mathrm{n}^{\circ}$ 152, 2008, p. 93-137.

CARDON Dominique, La démocratie Internet, Paris, Seuil, 2010.

CARDON Dominique et CASILLI Antonio, Qu'est-ce que le digital labor ?, Paris, Ina, 2015.

DUJARIER Marie-Anne, Le travail du consommateur, Paris, La Découverte, 2014.

FUCHS Christian, Digital Labor and Marx, New York, Routledge, 2014.

HIRSCHMANN Albert O., Exit. Voice. Loyalty : Défection et prise de parole, Bruxelles, Éd. de l'Université de Bruxelles, 2011.

KARPIK Lucien, L'économie des singularités, Paris, Gallimard, 2007.

Mogoutov Andreï, « Making Collaboration Networks Visible », LATOUR B. et WEIBEL P. (dir.), Making Things Public-Atmospheres of Democracy, Cambridge, MIT Press, 2005.

RIBAC François, «Les éditeurs ont perdu le monopole de la reproduction de musique à grande échelle », entretien pour Musique Info Hebdo, $\mathrm{n}^{\circ}$ 443, juillet 2007.

RIBAC François, «Ce que les usagers et Internet font à la prescription culturelle publique et à ses lieux : l'exemple de la musique en Ile de France ». Rapport de recherche pour le Programme Culture et Territoires en Île-de-France, Paris, 2010.

STIEGLER Bernard, De la misère symbolique, Paris, Flammarion, 2013.

TOSCHES Nick, Héros oubliés du rock'n'roll, les années sauvages du rock avant Elvis, traduit de l'anglais par Jean Marc MANDosio, Paris, Éditions Allia, 2000.

scHolz Trebor (ed.), Digital Labor, The Internet as Playground and Factory, New York, Routledge, 2013.

\section{NOTES}

1. Par exemple la plate-forme d'échanges Soulseek.

2. Ce que les usagers et Internet font à la prescription culturelle publique et à ses lieux : l'exemple de la musique en Ile de France». Étude financée par le programme interministériel « culture et territoire en Ile de France » et dont le rapport est disponible en ligne: http://rp.urbanisme.equipement.gouv.fr/puca/activites/rapport-usagersinternet-prescription-culturelle-publique.pdf

3. J'ai moi-même créé un blog consacré à l'enquête : http://www.blogs-et-prescriptionen-idf.net/ 
4. À ce sujet voir la notion de plateforme expressive dans ALLARD Laurence, «Émergence des cultures expressives, d'Internet au mobile », Mediamorphoses, nº 21, 2007, p. 19-25.

5. Blogroll : liste de liens pointant vers d'autres sites figurant généralement à droite ou à gauche de la page d'accueil d'un blog.

6. Les noms entourés d'une zone grise désignent des personnes dont l'activité a été étudiée sur plusieurs sites.

7. Ce classement en trois strates peut être rapproché de celui opéré par Dominique Cardon (CARDON Dominique, «Le désign de la visibilité Un essai de cartographie du web 2.0 ", Réseaux, $\mathrm{n}^{\circ} 152,2008$, p.104) qui dans son analyse des différentes façons dont les usagers du Web 2.0 dévoilent (ou pas) leur identité et leurs connexions parle respectivement de paravent (derrière lequel se cacheraient «mes » indépendants), de clair-obscur (les alternatifs) et de phare (les réseauteurs).

8. Sociétés qui à l'occasion de tournées de formations musicales les proposent à des salles de concert ou des festivals.

9. Haute Autorité pour la diffusion des Cuvres et la Protection des Droits sur Internet créée par la loi de 2009 et ayant pour objet de combattre le téléchargement illégal.

10. Logiciel permettant de repérer en ligne des débats et/ou des mots clés et d'établir des réseaux sémantiques. Pour cette étude le logiciel a notamment servi à associer différents moteurs de recherche et compiler leurs résultats.

11. Rip : transfert sous forme de fichiers numériques de disques vinyles.

12. Forgé par Andreï Mogoutov, le terme rézodience amalgame les mots audience et réseaux et est intimement lié à l'usage de logiciels qui recueillent des données en ligne. Voir Mogoutov Andreï, « Making Collaboration Networks Visible », LATOUR B. et WEIBEL P. (dir.), Making Things Public-Atmospheres of Democracy, Cambridge, MIT Press, 2005. Il signifie que l'on se propose d'analyser l'audience d'une entité en termes de réseaux et de connexions et que l'on va croiser des données quantitatives (par exemple le nombre de liens qui pointent vers un site) avec une analyse qualitative (par exemple l'analyse des composantes de ce réseau). On trouvera d'autres méthodes d'évaluation et de représentation des pratiques sur le Web dans les deux numéros (152 et 154) de la revue Réseaux consacrés au Web 2.0 dirigés par Dominique Cardon.

13. Le code source se présente sous la forme d'une suite de fichiers texte et de liens, des lignes de code où figurent les instructions permettant de mettre en page et d'ordonner un site. Les codes source des blogs sont accessibles à partir d'un menu figurant dans le navigateur que l'on utilise pour surfer sur la toile.

14. URL : adresse par laquelle on accède à un site.

15. Un constat qui recoupe les analyses des usages et de la construction de sa réputation sur MySpace (avant son déclin) par Jean-Samuel Beuscart et sur Flickr par Beuscart, Cardon, Pissard et Prieur. Voir BEUSCART Jean-Samuel, CARDon Dominique, PISSARD Nicolas, PRIEUR Christophe, «Pourquoi partager mes photos de vacances avec des inconnus, les usages de Flickr », Réseaux, n 54, 2009, p. 93-129 et BEUSCART Jean-Samuel, «Sociabilité en ligne, notoriété virtuelle et carrière artistique, les usages de MySpace par les musiciens autoproduits ", Réseaux, nº 152, 2008, p. 139-168.

16. Blog La fille du Rock (consulté le 8 septembre 2016). 
17. Sur ce phénomène: BEUSCART Jean-Samuel et MELLET Kevin, «La conversion de la notoriété en ligne. Une étude des trajectoires de vidéastes pro-am », Terrains et Travaux , vol. 26, nº 1, 2015, p 83 -104.

18. Coûts d'impression ou de diffusion, masse salariale, achat des flux d'infos, nécessité de s'assurer d'un public nombreux, recherches d'annonceurs, distribution, accès aux œuvres et aux informations etc.

19. KARPIK Lucien, L'économie des singularités, Paris, Gallimard, 2007, p. 68-81.

20. Selon Karpik, l'ensemble de ces instances constitue un « régime de coordination » ( Ibid., p. 137-156).

21. On peut même penser qu'un nombre significatif de personnes continuent justement à accorder du crédit aux « prescriptions traditionnelles » car elles se méfient du Web et de sa position de plus en plus dominante (exit).

22. Voir par exemple BOUQUILLON Philippe et MATTHEWS Jacob T., Le Web collaboratif. Mutations des industries de la culture et de la communication, Grenoble, Presses universitaires de Grenoble, 2010 ; scholz Trebor (ed.), Digital Labor, The Internet as Playground and Factory, New York, Routledge, 2013 ; FUCHS Christian, Digital Labor and Marx, New York, Routledge, 2014 ; DUJARIER Marie-Anne, Le travail du consommateur, Paris, La Découverte, 2014 ; CARDON Dominique et CASILLI Antonio, Qu'est-ce que le digital labor ?, Paris, Ina, 2015. Merci à Paola Sedda pour ses précieux conseils bibliographiques.

\section{RÉSUMÉS}

La numérisation de la musique enregistrée puis l'apparition des réseaux de peer to peer ont fortement affecté l'économie de la musique, ses métiers, son architecture, sa division du travail. Ce phénomène majeur s'est accompagné d'une autre mutation non moins importante. À la faveur de la mise en ligne des outils du Web 2.0 (blogs, sites de partages, réseaux sociaux), une critique amateur(e) s'est constituée et a su trouver son public. Cette blogosphère musicale a développé de nouvelles façons de faire partager (l'amour) de la musique, d'émettre des préconisations, de promouvoir son activité. Une forme de recommandation bénévole qui a désormais pris sa place dans le monde musical aux côtés de la prescription professionnelle.

\section{INDEX}

Mots-clés : Prescription culturelle, économie de la musique, amateurs, musique et Internet

\section{AUTEUR}

\section{FRANÇOIS RIBAC}

François Ribac est compositeur de théâtre musical, maître de conférences à l'université de Dijon, laboratoire Cimeos. Ses recherches portent sur les musiques populaires, la reproduction sonore, 
la prescription culturelle. Il est responsable du projet de recherche ASMA (Arts de la Scène et Musique dans l'Anthropocène) 2016-2019. Il est l'auteur de sept opéras et de musiques pour la télévision. Dernier ouvrage publié : La Fabrique de la programmation culturelle (La Dispute, 2017). 